\title{
Avian Biodiversity of Newly Established Bunjosa Game Reserve, District Poonch, Azad Jammu and Kashmir, Pakistan: Implications for Conservation
}

\author{
Muhammad Umar khan ${ }^{1}$, Nausheen Irshad ${ }^{1}$, Muhammad Kabir ${ }^{2}$ and Muhammad Naeem Awan ${ }^{3 *}$ \\ ${ }^{1}$ Department of Zoology, University of Poonch, Pakistan \\ ${ }^{2}$ Department of Forestry and Wildlife Management, University of Haripur, Pakistan \\ ${ }^{3}$ Himalayan Nature Conservation Foundation, Pakistan
}

Submission: January 16, 2019; Published: January 29, 2019

*Corresponding author: Muhammad Naeem Awan, Himalayan Nature Conservation Foundation, Muzaffarabad, Azad Jammu and Kashmir, Pakistan

\begin{abstract}
Bunjosa game reserve lies in district Poonch of Azad Jammu and Kashmir, Pakistan. This game reserve is newly established so, avian fauna of this area was unexplored yet, so the present study was undertaken to determine the avian biodiversity, residential status and ecological threats. The findings of the bird's surveys undertaken from October 2015 to November 2016. A total of $n=3137$ birds belonging to 108 species, 25 families and 11 orders were identified with highest numbers in summer $(\mathrm{n}=1148)$ and lowest in winter $(\mathrm{n}=415)$. The seasonal Shannon Wiener diversity index was highest in summer (4.3), followed by autumn (3.99), winter (3.64) and spring (3.58), likewise the family wise diversity index was maximum for family Corvidae (0.26) and minimum for Remizidae, Phasianidae and Alcedinidae (0.006) respectively. The research findings revealed that habitat fragmentation and clearance through extensive deforestation, forest fires, livestock grazing, and collection of fire woods are recorded as the major threats to the avifauna. Development of conservation and management plan will be helpful in protecting the avian biodiversity and ecosystem of the game reserve.
\end{abstract}

Keywords: Abundance; Birds; Bunjosa; Diversity; Habitat; Richness

\section{Introduction}

Birds are among the most popular indicators of environmental changes and have been used to assess the environment throughout the history as bio-monitors. The changes in their population, reproductive ability and behavior patterns have most often been used to study the long-term effects of habitat fragmentation. Hence, they are the good indicators of ecological status of any specified ecosystem [1]. They also act as scavengers and pollinating agents and play a prominent role in the seed's dispersal as well as in religion and culture. Besides they are also a main source of food for mankind and are very familiar to man since centuries [2].

It is an unpleasant fact that global diversity of birds is decreasing at an alarming rate primarily due to unhealthy anthropogenic activities and climatic changes [3-5]. The common threats associated with bird species today are destruction and degradation of their habitat which affects more than 93 percent bird species worldwide. Habitat loss is the most critical reason of destruction of biodiversity throughout the world [6]. Other important threats are rapid spread of agriculture, over hunting, deforestation, invasive species and climatic changes.
The diversity and abundance of avian fauna in a specific habitat could serve as a valuable indicator of the ecological status of that habitat. Therefore, the ornithological data might provide a soul basis to assess the effects of environmental changes on biodiversity. Bird indicators play a key role to form an important component of sets of indicators for biodiversity and habitats. The present climatic changes also had harmful effects in bird life and ecological balance. So, it is essential to save the bird species from the threats in order to maintain the biodiversity. By keeping this in mind the current study was planned to explore the avian diversity and to highlight major threats to the avian fauna of Bunjosa game reserve.

\section{Materials and Methods}

\section{Study area}

Bunjosa game reserve (BGR, 3348`35.92”N, 7348`58.80”E) is a protected area located $18 \mathrm{~km}$ north of Rawalakot Azad Kashmir, Pakistan in foot hills of Himalayan region, covering almost 1385 hectares area at 1777.5-1966.8m elevation (Figure 1). There is a magnificent artificial lake alongside with reserve which is a permanent source of water for the wildlife particularly the avian 
fauna of this area. The lake is surrounded by dense pine forests all around and they provide an ideal habitat for several bird's species. The forest type is moist temperate with dominant plants vegetation of Pinus roxburjii, Pinus wallichiana, Cidrus deodara and Berberies lyceum, while the most dominant mammalian fauna is Rhesus monkey (Macaca mulatta), Common leopard (Panthera pardus), Black bear (Ursus amricanus), Red fox (Vulpes vulpes griffithi), Palm civet (Paguma larvata), Porcupine (Hystric indica), Squirrel (Suiarus carolinensis), Wild pig (Sus scrufa) and Wild dog (Canis lupis). The area lies under the influence of monsoon with March and April being the wettest months and is exposed to heavy snowfall during the winter. Maximum temperature is usually recorded to be $30^{\circ} \mathrm{C}$ while minimum is below $0^{\circ} \mathrm{C}$.

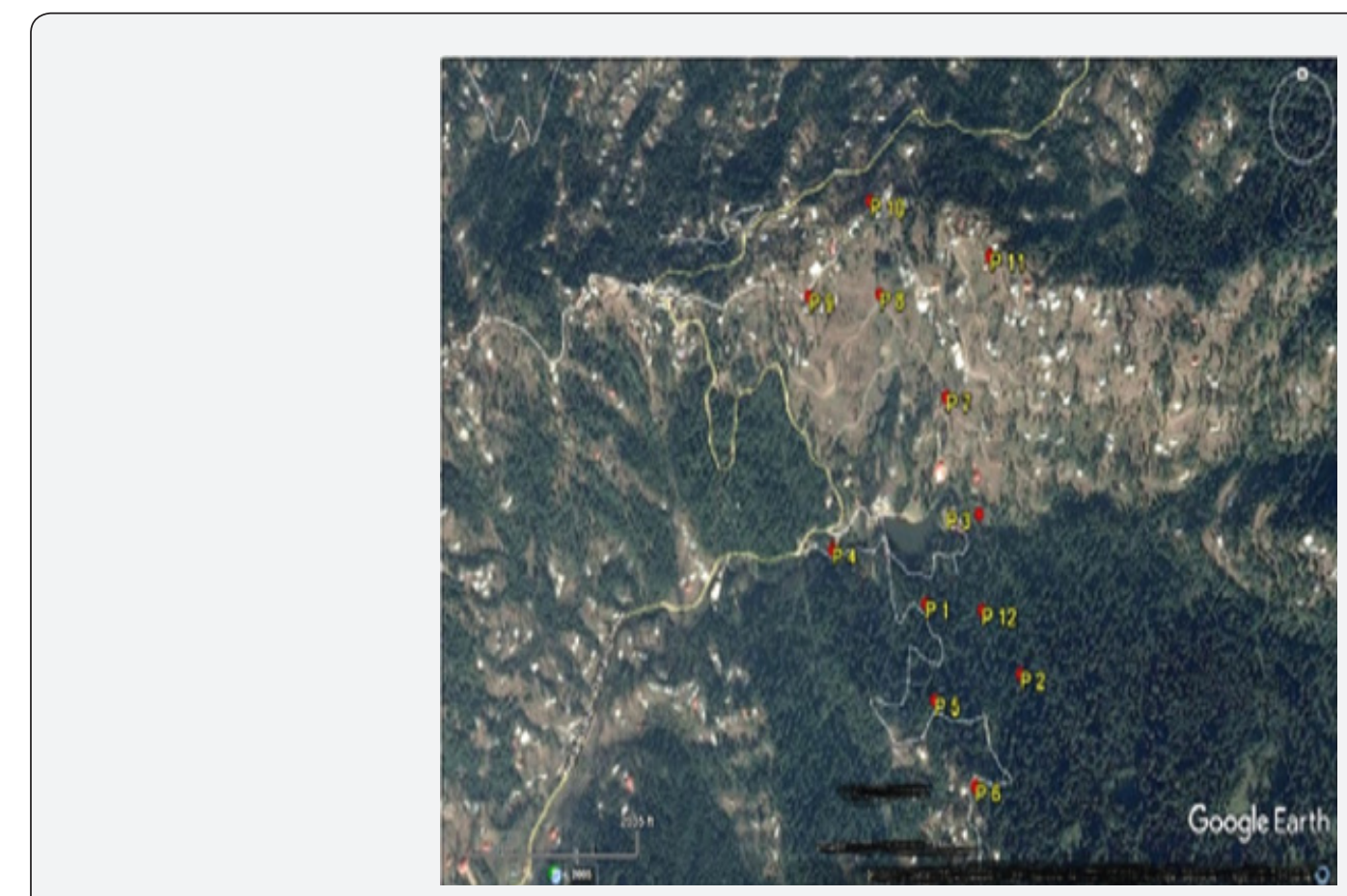

Figure 1: Satellite map of Bunjosa game reserve, showing different selected survey points in the study area.

\section{Methodology}

The present study was carried out from October 2015 to November 2016 to record bird's diversity and related conservation issues of Bunjosa game reserve by using point count surveys with unlimited radius $[7,8]$. Twelve survey points were positioned randomly approximately $0.5 \mathrm{Km}$ apart between 1777.5 and $1966.8 \mathrm{~m}$ elevation which were representative of the altitudinal range and habitat covered by the reserve. Each of the twelve survey points were surveyed twice per month, in morning from 7.00am to $10.00 \mathrm{am}$ and in evening from $4.00 \mathrm{pm}$ to $6.00 \mathrm{pm}$ in the first week of each month. In total each plot was surveyed 24 times so the total survey efforts were 288. The bird fauna was observed using Binocular (12x a50xs) and identified using keys given by Grimmett [9] \& Mirza [10].

Bird survey data were supplemented by information gathered from interviewed with local people about the importance of bird species in our environment and the impact of anthropogenic activities on the birds and their habitat. A total of 200 people was interviewed, and they were selected randomly from the village around the reserve.

The Shannon Wiener diversity index was analyzed for calculating relative abundance, species richness and encounter rate using following statistics.
$H^{\prime}=-[\Sigma P i \ln P i]$ Where $\mathrm{H}^{\prime}$ is the diversity index,

$\mathrm{Pi}$ is the relative abundance (proportion of species relative to total number of species) and Log $\ln \mathrm{Pi}$ is the natural logarithm of this proportion [11]

Relative abundance was calculated as

Relative abundance $=P i=n i / N$

Where ni is the abundance (Number of individuals of a species) and $\mathrm{N}$ is the total population of birds. Species encounter rates were calculated based on number of birds recorded from all point surveyed and presented as numbers per hundred-point counts. Encounter rates were converted into ordinal categories of abundance as $\leq 5$ birds per 100point count(abundant), 5.1-10(Uncommon), 10.1-20 (frequent), 20.1-40 (Common) and $>40$ (abundant) [12].

\section{Results and Discussion}

During the study $\mathrm{n}=108$ bird species belonging to $\mathrm{n}=12$ orders and $n=27$ families were identified. Of these $n=52$ species were resident, $\mathrm{n}=39$ were summer migrant, $\mathrm{n}=15$ were winter migrant and $n=2$ were passage migrant. Three $(n=3)$ species were marked as abundant, $\mathrm{n}=8$ as common, $\mathrm{n}=9$ as frequent, $\mathrm{n}$ $=45$ as uncommon and $n=43$ as Rare (Table 1) [8]. The highest 
number of species was recorded in the family Turdidae of order Turdiformes while Shanon-Wiener Diversity Index was recorded (4.3) in summer followed by (3.99) in autumn, (3.64) in winter and (3.58) in spring respectively.

The Relative Abundance of some common bird species was observed and presented in Table 1 that predicts prominent birds of study area. The relative abundance recorded for Common myna (0.072), Yellow billed blue magpie (0.015), Himalayan laughing thrush (0.031), Yellow and Grey wagtails (0.006), Great tit
(0.008), Yellow cheeked tit (0.004), Crested black tit (0.008), Red headed long tail tit (0.03) and Green backed tit (0.025). Mausad et al. [13] during the survey of Chinari district Hattian, Pakistan, found relative abundance of Common myna (0.055), Himalayan laughing thrush (0.002) and Yellow billed blue magpie (0.015). Awan et al. [14] recorded relative abundance of Yellow and Grey wagtails ( 0.16 percent and 0.24 percent) in Salkhala game reserve (SGR) Pakistan, whereas Awan et al. [15] reported five species of Tits from Machiara national park, Pakistan.

Table 1: Species encounter rate as per 100point counts. Abundance (Ordinal scale) A (Abundant), C (Common), F (Frequent), U (Uncommon), R (Rare). Status scale (Month observed) R (Resident), S (Summer migrant), W (Winter migrant), P (Passage migrant) L (Local movement) Awan et al. [8].

\begin{tabular}{|c|c|c|c|c|c|c|}
\hline Species & Scientific Name & $\begin{array}{l}\text { Encounter } \\
\text { Rate (ER) }\end{array}$ & $\begin{array}{l}\text { Abundance } \\
\text { (n) }\end{array}$ & $\begin{array}{l}\text { Relative Abun- } \\
\text { dance (p) }\end{array}$ & Status (Months Observed) & Altitude (m) \\
\hline $\begin{array}{l}\text { Yellow-billed blue } \\
\text { magpie }\end{array}$ & Urocissa flavirostris & 72.5 & A (209) & 0.066 & $\begin{array}{l}\text { R, L (Feb-Mar, Apr- May, Jun- } \\
\text { Jul, Aug- Sep, Oct.) }\end{array}$ & 1777.5- 1966.8 \\
\hline Lanceolated jay & Garrulus lanceolatus & 16.3 & $\mathrm{~F}(47)$ & 0.014 & $\begin{array}{l}\text { R, L (Mar- Apr, May- Jun, Jul- } \\
\text { Aug, Sep-Oct, Nev) }\end{array}$ & $1777.5-1966.8$ \\
\hline $\begin{array}{l}\text { Himalayan jungle } \\
\text { crow }\end{array}$ & Corrus macrorhynchous & 31.2 & $\mathrm{C}(90)$ & 0.028 & $\begin{array}{l}\text { R, L (Feb- Mar, Apr- May, Jun- } \\
\text { Jul, Aug- Sep,Oct -Nev) }\end{array}$ & $1777.5-1966.8$ \\
\hline House crow & Corvus splendens & 10.4 & $\mathrm{~F}(30)$ & 0.009 & $\begin{array}{l}\text { R, L (Feb- May, Jun-Jul, Aug- } \\
\text { Sep, Oct- Nev) }\end{array}$ & $1777.5-1934.7$ \\
\hline Rufous tree pie & Dendrocita Vagabunda & 0.1 & $\mathrm{R}(9)$ & 0.002 & W (Feb-Sep, Oct- Nev) & $1777.5-1911.7$ \\
\hline Yellow billed caugh & Pyrrhocorax graculus & 0.1 & $\mathrm{R}(11)$ & 0.003 & W (Sep-Oct) & $1834.8-1966.8$ \\
\hline Carrion crow & Corvus corone & 0.1 & $\mathrm{R}(11)$ & 0.003 & $\begin{array}{l}\text { R (Feb-May, Jun-Jul, Aug- } \\
\text { Nev) }\end{array}$ & 1789.7-1966.8 \\
\hline Common myna & Acredotheres tristis & 79.1 & $A(228)$ & 0.072 & $\begin{array}{l}\text { R, L (Feb-Nev, Mar- Apr, May- } \\
\text { Jun, Jul- Aug, Sep-Oct) }\end{array}$ & $1777.5-1888.2$ \\
\hline Black bulbul & $\begin{array}{l}\text { Hypsipetes madagas- } \\
\text { cariensis }\end{array}$ & 27 & $\mathrm{C}(78)$ & 0.024 & $\begin{array}{l}\text { R, L (Feb- Mar, Apr- May, Jun- } \\
\text { Jul, Aug-Sep, Oct- Nev) }\end{array}$ & $1777.5-1966.8$ \\
\hline Red vented bulbul & Pycnonotus cafer & 9 & $\mathrm{U}(26)$ & 0.008 & S (May- Jun, Jul-Aug, Sep) & 1848.9-1934.7 \\
\hline White cheeked bulbul & Pycnonotus leucogenys & 2.7 & $\mathrm{R}(8)$ & 0.015 & $\begin{array}{l}\text { R (Feb-Mar, Apr-Sep, Oct- } \\
\text { Nev) }\end{array}$ & 1848.6-1911.7 \\
\hline Ashy drongo & Dicrurus leucophaeus & 9.3 & $\mathrm{U}(27)$ & 0.008 & $\begin{array}{l}\text { R, L (Mar- Apr, May- Jun, Jul- } \\
\text { Aug, Sep-Oct) }\end{array}$ & 1834.5-1911.7 \\
\hline Black drongo & Dicrurus macrocercus & 4.1 & $\mathrm{R}(12)$ & 0.004 & S (May- Jun, Jul- Aug) & 1841.8-1934.7 \\
\hline House sparrow & Passer domesticus & 17 & $\mathrm{~F}(49)$ & 0.015 & $\begin{array}{l}\text { R, L (Feb-Mar, Apr- May, Jun- } \\
\text { Jul, Aug-Sep, Oct -Nev) }\end{array}$ & 1777.5-1911.7 \\
\hline $\begin{array}{l}\text { Cinnamon tree } \\
\text { sparrow }\end{array}$ & Passer rutilans & 7.9 & $\mathrm{U}(23)$ & 0.007 & $\begin{array}{l}\text { R, L (May- Jun, Jul- Aug, } \\
\text { Sep-Oct) }\end{array}$ & 1777.5-1911.7 \\
\hline Scrub sparrow & Passer moabiticus & 5.9 & $\mathrm{U}(17)$ & 0.005 & S (May- Jun, Jul- Aug) & 1777.7-1911.7 \\
\hline $\begin{array}{l}\text { Eurasian tree spar- } \\
\text { row }\end{array}$ & Passer montanus & 3.8 & $\mathrm{R}(11)$ & 0.003 & $\begin{array}{l}\text { R (May- Jun, Jul- Aug, Sep- } \\
\text { Oct) }\end{array}$ & $1789.7-1805.0$ \\
\hline $\begin{array}{l}\text { Migratory house } \\
\text { sparrow }\end{array}$ & Passer domesticus & 5.2 & $\mathrm{U}(15)$ & 0.004 & S (May- Jun, Jul- Aug) & 1777.5-1911.7 \\
\hline $\begin{array}{l}\text { Chestnut shouldered } \\
\text { petronia }\end{array}$ & Petronia xanthocollis & 3.4 & $\mathrm{R}(10)$ & 0.003 & S (May- Jun, Jul- Aug) & $1777.5-1888.2$ \\
\hline Grey headed sparrow & Passer griseus & 7.9 & $\mathrm{U}(23)$ & 0.007 & $\begin{array}{l}\text { R, L (Feb- May, Jun-Jul, Aug- } \\
\text { Oct, Nev) }\end{array}$ & 1789.7-1911.7 \\
\hline Common swallow & Hirundo rustica & 22.9 & $C(66)$ & 0.021 & $\begin{array}{l}\text { S (Mar- Apr, May-Jun, Jul- } \\
\text { Aug) }\end{array}$ & $1777.5-1888.2$ \\
\hline $\begin{array}{l}\text { Blue headed yellow } \\
\text { wagtail }\end{array}$ & Motacilla flava bema & 6.9 & $\mathrm{U}(20)$ & 0.006 & $\begin{array}{l}\text { R, L (Mar- Apr, May- Jun, Jul- } \\
\text { Aug, Sep-Oct) }\end{array}$ & $1777.5-1841.9$ \\
\hline Grey wagtail & Motacilla cinerea & 6.5 & U (19) & 0.006 & $\begin{array}{l}\text { R, L (Feb-Mar, Apr-May, Jun- } \\
\text { Jul, Aug-Sep, Oct-Nev) }\end{array}$ & $1777.5-1841.9$ \\
\hline
\end{tabular}




\begin{tabular}{|c|c|c|c|c|c|c|}
\hline Long-billed pipet & Anthus similis & 5.9 & U (17) & 0.005 & $\begin{array}{l}\text { R (Mar-Apr, May, Jun, Jul- } \\
\text { Aug, Sep-Oct) }\end{array}$ & $1777.5-1848.6$ \\
\hline Tree pipet & $\begin{array}{l}\text { Anthus trivialis bar- } \\
\text { ingtoni }\end{array}$ & 3.1 & $\mathrm{R}(9)$ & 0.002 & S (May- Jun, Jul- Aug) & $1777.5-1888.2$ \\
\hline Twany pipet & Anthus campestris & 3.8 & $\mathrm{R}(11)$ & 0.003 & S (May- Jun, Jul- Aug) & $1777.5-1848.6$ \\
\hline Water pipet & Anthus spinoletta & 3.8 & $\mathrm{R}(11)$ & 0.003 & $\begin{array}{l}\text { R (Feb-Mar, Apr-May, Jun-Jul, } \\
\text { Aug- Nev) }\end{array}$ & $1777.5-1841.9$ \\
\hline $\begin{array}{l}\text { Himalayan laughing } \\
\text { thrush }\end{array}$ & Garrulax lineatus & 34.7 & $C(100)$ & 0.031 & $\begin{array}{l}\text { R, L (Feb- Mar, Apr- May, Jun- } \\
\text { Jul, Aug- Sep, Oct- Nev) }\end{array}$ & $1777.5-1966.8$ \\
\hline $\begin{array}{l}\text { Sliver eared laughing } \\
\text { thrush }\end{array}$ & $\begin{array}{l}\text { Trochalopteron mela- } \\
\text { nostigma }\end{array}$ & 1 & $\mathrm{R}(3)$ & 0.0009 & W (Sep-Oct) & $1777.5-1966.8$ \\
\hline Red billed leiothrix & Leiothrix lutea & 8.7 & $\mathrm{U}(35)$ & 0.011 & $\begin{array}{l}\text { R (Feb-Mar, Apr-May, Jun-Jul, } \\
\text { Aug-Sep, Oct- Nev) }\end{array}$ & $1777.5-1966.8$ \\
\hline Haw finch & $\begin{array}{c}\text { Coccothraustes cocco- } \\
\text { thraustes }\end{array}$ & 4.5 & $\mathrm{R}(13)$ & 0.004 & $\begin{array}{l}\text { R p- ul- May- Jun-Jul- Oct S } \\
\text { (May- Jun, Jul-Aug) }\end{array}$ & 1856.8-1934.7 \\
\hline $\begin{array}{l}\text { Himalayan green } \\
\text { finch }\end{array}$ & Carduelis spinoides & 9.4 & $\mathrm{U}(27)$ & 0.008 & R (Feb-Nev, Sep- Oct) & 1848.6-1934.7 \\
\hline $\begin{array}{l}\text { Red munia or Ava- } \\
\text { davat }\end{array}$ & Estrilda amandva & 6.6 & $\mathrm{U}(19)$ & 0.006 & $\begin{array}{l}\text { R (May- Jun, Jul-Aug, Sep- } \\
\text { Oct) }\end{array}$ & 1805.0-1911.7 \\
\hline Twite & Carduelis flavirostris & 3.5 & $\mathrm{R}(10)$ & 0.003 & $\begin{array}{l}\text { R (May- Jun, Jul- Aug, Sep- } \\
\text { Oct) }\end{array}$ & $1856.8-1934.7$ \\
\hline Scaly breasted munia & Lonchura punctulata & 15.9 & $F(46)$ & 0.014 & $\begin{array}{l}\text { R (May- Jun, Jul- Aug, Sep- } \\
\text { Oct) }\end{array}$ & $1777.5-1888.2$ \\
\hline $\begin{array}{l}\text { Chestnut eared } \\
\text { bunting }\end{array}$ & Emberiza fucata & 4.2 & $\mathrm{R}(12)$ & 0.003 & $\begin{array}{l}\text { R (Feb-Mar, Apr-Sep, Oct } \\
\text {-Nev) }\end{array}$ & 1789.7-1888.2 \\
\hline Red headed bunting & Emberiza bruniceps & 6.9 & $\mathrm{U}(20)$ & 0.006 & R (Feb- Sep, Oct- Nev) & 1841.9-1966.8 \\
\hline Eurasian linnet & Carduelis cannabina & 7.9 & $\mathrm{U}(23)$ & 0.007 & S (May- Jun, Jul- Aug) & $1841.9-1934.7$ \\
\hline Red backed shrike & Lanius collurio & 5.9 & $\mathrm{U}(17)$ & 0.005 & W (Sep-Oct) & 1841.9-1966.8 \\
\hline Common rose finch & Carpodacus erythrinus & 4.5 & $\mathrm{R}(13)$ & 0.004 & S (May- Jun, Jul- Aug) & 1841.9-1934.7 \\
\hline $\begin{array}{l}\text { Thick- billed flower- } \\
\text { pecker }\end{array}$ & Dicaeum agile & 33.3 & C (96) & 0.03 & $\begin{array}{l}\text { R, L (Feb-Mar, Apr-May, Jun- } \\
\text { Jul, Aug-Sep, Oct-Nev) }\end{array}$ & $1777.5-1966.8$ \\
\hline Oriental white eye & Zosterops Palpebrosa & 14.9 & $F(43)$ & 0.013 & $\begin{array}{l}\text { R, L (May- Jun, Jul- Aug, } \\
\text { Sep-Oct) }\end{array}$ & $1777.5-1966.8$ \\
\hline $\begin{array}{l}\text { White capped red- } \\
\text { start }\end{array}$ & $\begin{array}{l}\text { Chaimarromis leuco- } \\
\text { cephalous }\end{array}$ & 4.2 & $\mathrm{R}(12)$ & 0.003 & R (Feb-Sep, Oct- Nev) & $1777.5-1789.7$ \\
\hline $\begin{array}{l}\text { Stone chat or Collard } \\
\text { indian bush chat }\end{array}$ & Saxicola torquata & 6.6 & U (19) & 0.006 & $\begin{array}{l}\text { R (May- Jun, Jul- Aug, Sep- } \\
\text { Oct) }\end{array}$ & 1805.0-1911.7 \\
\hline $\begin{array}{l}\text { Grey winged black } \\
\text { bird }\end{array}$ & Turdus boulboul & 9 & U (26) & 0.008 & $\begin{array}{l}\text { R (Feb-Nev, Mar- Apr, Sep- } \\
\text { Oct) }\end{array}$ & $1777.5-1966.8$ \\
\hline Indian blue robin & Luscinia brunnea & 7.3 & $\mathrm{U}(21)$ & 0.006 & $\begin{array}{l}\text { R (May- Jun, Jul- Aug, Sep- } \\
\text { Oct) }\end{array}$ & 1789.7-1911.7 \\
\hline Blue capped redstart & $\begin{array}{l}\text { Phoenicurus caeruleo- } \\
\text { cephalus }\end{array}$ & 3.1 & $\mathrm{R}(9)$ & 0.002 & $\begin{array}{l}\text { R (Feb- Mar, Apr-Sep, Oct- } \\
\text { Nev) }\end{array}$ & 1789.7-1934.7 \\
\hline Grey bush chat & Saxicola ferrea & 13.5 & F (39) & 0.012 & $\begin{array}{l}\text { R, L (Mar- Apr, May- Jun, Jul- } \\
\text { Aug, Sep-Oct) }\end{array}$ & $1789.7-1966.8$ \\
\hline Blue whistling thrush & Myiophoneus caeruleus & 10.4 & $\mathrm{~F}(30)$ & 0.009 & $\begin{array}{l}\text { R (Feb-Mar, Apr-Sep, Oct- } \\
\text { Nev) }\end{array}$ & $1777.5-1888.2$ \\
\hline Blue rock thrush & Monticola solitaries & 2.4 & $\mathrm{R}(7)$ & 0.002 & W (Sep-Oct) & $1841.9-1966.8$ \\
\hline Hum`s wheatear & Oenanthe alboniger & 4.5 & $\mathrm{R}(13)$ & 0.004 & $\begin{array}{l}\text { R (Feb-May, Jun-Jul, Aug-Sep, } \\
\text { Oct- Nev) }\end{array}$ & $1789.7-1888.2$ \\
\hline Spotted forktail & Enicurus scouleri & 1.7 & $\mathrm{R}(5)$ & 0.001 & W (Feb-Sep, Oct) & $1777.5-1789.7$ \\
\hline $\begin{array}{l}\text { Rufous tailed } \\
\text { wheatear }\end{array}$ & $\begin{array}{l}\text { Oenanthe xanthoprym- } \\
n a\end{array}$ & 3.8 & $\mathrm{R}(11)$ & 0.003 & $\begin{array}{l}\text { R (Feb-May, Jun-Jul, Aug- } \\
\text { Nev) }\end{array}$ & 1876.6-1911.7 \\
\hline Pied bush chat & Saxicola caprata & 5.2 & $\mathrm{U}(15)$ & 0.004 & $\begin{array}{l}\text { R (Feb-May, Jun-Jul, Aug- } \\
\text { Nev) }\end{array}$ & $1834.8-1966.8$ \\
\hline
\end{tabular}


International Journal of Environmental Sciences \& Natural Resources

\begin{tabular}{|c|c|c|c|c|c|c|}
\hline Thickell,s thrush & Turdus unicolor & 3.1 & $\mathrm{R}(9)$ & 0.002 & W (Feb-Nev) & $1834.8-1966.8$ \\
\hline Chestnut thrush & Turdus rubrocanus & 5.5 & $\mathrm{U}(16)$ & 0.005 & W (Feb-Nev) & $1841.5-1876.8$ \\
\hline Variable wheatear & Onanthe picata & 4.2 & $\mathrm{R}(12)$ & 0.004 & W (Feb-Nev, Oct) & 1841.5-1876.8 \\
\hline Greenish warbler & $\begin{array}{l}\text { Phylloscopus torchiloi- } \\
\text { des }\end{array}$ & 3.1 & $\mathrm{R}(9)$ & 0.002 & S (May-Jun, Jul-Aug) & $1777.5-1789.7$ \\
\hline Brook,s leaf warbler & Phylloscopus subviridis & 7.6 & $\mathrm{U}(22)$ & 0.007 & R (Apr-May, Jun-Jul, Aug) & 1805.0-1911.7 \\
\hline $\begin{array}{l}\text { Yellow eyed flycatch- } \\
\text { er warbler }\end{array}$ & Seicercus burkii & 5.2 & $\mathrm{U}(15)$ & 0.004 & $\begin{array}{l}\text { R (Mar- Apr, May- Jun, Jul- } \\
\text { Aug, Sep-Oct) }\end{array}$ & 1848.6-1888.2 \\
\hline Common chiffchaff & Phyllocopus collybita & 3.1 & $\mathrm{R}(9)$ & 0.002 & P (Mar- Apr) & 1841.9-1934.7 \\
\hline Mountain chiffchaff & Phyllocopus sindianus & 3.1 & $\mathrm{R}(9)$ & 0.002 & S (Apr-May, Jun-Jul, Aug) & 1848.6-1934.7 \\
\hline $\begin{array}{l}\text { Grey headed flycatch- } \\
\text { er warbler }\end{array}$ & Seicercus xanthoschistos & 34.7 & $C(100)$ & 0.031 & $\begin{array}{l}\text { R, L (Feb-Mar, Apr-May, Jun- } \\
\text { Jul, Aug- Sep, Oct- Nev) }\end{array}$ & $1777.5-1966.8$ \\
\hline Lesser whitethroat & Sylvia curruca & 4.2 & $\mathrm{R}(12)$ & 0.003 & S (Mar- Apr, Sep) & $1777.5-1934.7$ \\
\hline Whistler warbler & Seicercus whistleri & 3.5 & $\mathrm{R}(10)$ & 0.003 & $\begin{array}{l}\text { R (Feb-Mar, Apr-May, Jun-Jul, } \\
\text { Aug- Nev) }\end{array}$ & 1841.9-1966.8 \\
\hline $\begin{array}{l}\text { Moustached sadge } \\
\text { warbler }\end{array}$ & $\begin{array}{l}\text { Acrocephalus mela- } \\
\text { nopogon }\end{array}$ & 6.9 & $\mathrm{U}(20)$ & 0.006 & $\begin{array}{l}\text { S (Mar- Apr, May- Jun, Jul- } \\
\text { Aug) }\end{array}$ & $1777.5-1934.7$ \\
\hline Greater whitethroat & Sylvia communis & 5.5 & $\mathrm{U}(16)$ & 0.005 & S (May- Jun, Jul- Aug, Sep) & 1805.0-1876.6 \\
\hline Black crested tit & Parus rufonuchalis & 4.5 & $\mathrm{R}(13)$ & 0.004 & S (May- Jun, Jul- Aug) & $1777.5-1966.8$ \\
\hline Crested black tit & Parus melanolophus & 8.7 & $\mathrm{U}(25)$ & 0.008 & S (Jul-Aug, Sep-Oct) & $1777.5-1911.7$ \\
\hline Green backed tit & Parus monticolus & 27.4 & C (79) & 0.025 & $\begin{array}{l}\text { R, L (Feb-Mar, Apr- May, Jun- } \\
\text { Jul, Aug-Sep, Oct- Nev) }\end{array}$ & $1777.5-1966.8$ \\
\hline Yellow cheeked tit & Parus xanthogenys & 5.2 & $\mathrm{U}(15)$ & 0.004 & S (Apr-May, Jun-Jul) & $1777.5-1876.6$ \\
\hline Great tit & Parus major & 9 & $\mathrm{U}(26)$ & 0.008 & S (Jul- Aug, Sep) & 1805.0-1934.7 \\
\hline $\begin{array}{l}\text { Red headed long } \\
\text { tailed tit }\end{array}$ & Aigithalus concinnus & 33.3 & C (96) & 0.03 & $\begin{array}{l}\text { R, L (Feb- Mar, Apr- May, Jun- } \\
\text { Jul, Aug- Nev) }\end{array}$ & $1777.5-1876.6$ \\
\hline Penduline tit & Remiz pendulinus & 1.7 & $\mathrm{R}(5)$ & 0.001 & S (May- Jun, Jul- Aug) & $1777.5-1841.9$ \\
\hline Fire capped tit & $\begin{array}{l}\text { Cephalapyrus flammi- } \\
\text { ceps }\end{array}$ & 4.5 & $\mathrm{R}(13)$ & 0.004 & S (May- Jun, Jul- Aug) & $1777.5-1966.8$ \\
\hline $\begin{array}{l}\text { Ultramarine fly- } \\
\text { catcher }\end{array}$ & Ficedula Superciliaris & 4.2 & $\mathrm{R}(12)$ & 0.003 & S (Mar- Apr, May) & 1848.6-1934.7 \\
\hline Brown flycatcher & Muscicapa latirostris & 4.2 & $\mathrm{R}(12)$ & 0.003 & S (Jun-Jul, Aug) & 1841.9-1934.7 \\
\hline Slaty blue flycatcher & Ficedula tricolor & 4.5 & $\mathrm{R}(13)$ & 0.004 & S (Apr-May, Jun-Jul, Aug) & $1777.5-1911.7$ \\
\hline Verditer flycatcher & Muscicapa thalassina & 6.2 & $\mathrm{U}(18)$ & 0.005 & $\begin{array}{l}\text { S (Mar- Apr, May- Jun, Jul- } \\
\text { Aug) }\end{array}$ & $1789.7-1876.6$ \\
\hline Rufous billed niltava & Niltava sundara & 6.9 & $\mathrm{U}(20)$ & 0.006 & $\begin{array}{c}\text { R, L (Mar- Apr, May- Jun, Jul- } \\
\text { Aug, Sep-Oct) }\end{array}$ & $1777.5-1876.6$ \\
\hline Rufous tail flycatcher & Muscicapa ruficauda & 5.5 & $\mathrm{U}(16)$ & 0.005 & S (May- Jun, Jul- Aug, Sep) & $1789.7-1934.7$ \\
\hline $\begin{array}{l}\text { Blue throated fly- } \\
\text { catcher }\end{array}$ & Cyornis rubeculoides & 4.5 & $\mathrm{R}(13)$ & 0.004 & P (Mar- Apr) & $1789.7-1848.6$ \\
\hline Dark sided flycatcher & Muscicapa sibirica & 6.2 & $\mathrm{U}(18)$ & 0.006 & W (Sep-Oct) & $1789.7-1934.7$ \\
\hline $\begin{array}{l}\text { Grey headed canary } \\
\text { flycatcher }\end{array}$ & Culicicapa ceylonensis & 6.2 & $\mathrm{U}(18)$ & 0.006 & S (Jun-Jul, Aug) & $1876.6-1934.7$ \\
\hline $\begin{array}{l}\text { Red breasted fly- } \\
\text { catcher }\end{array}$ & Ficedula parva & 2.8 & $\mathrm{R}(8)$ & 0.002 & S (Jun-Jul, Aug- Sep) & 1777.5-1841.9 \\
\hline $\begin{array}{l}\text { Thickell,s blue fly- } \\
\text { catcher }\end{array}$ & Cyornis tickelliae & 7.6 & $\mathrm{U}(22)$ & 0.007 & S (Jun-Jul, Aug) & $1777.5-1876.6$ \\
\hline $\begin{array}{l}\text { Asian paradise fly- } \\
\text { catcher }\end{array}$ & Terpsiphone paradise & 2.4 & $\mathrm{R}(7)$ & 0.002 & W (Sep-Oct) & $1777.5-1789.7$ \\
\hline Black naped monarch & Monarcha azuerea & 4.2 & $\mathrm{R}(12)$ & 0.004 & S (May- Jun, Jul- Aug) & $1777.5-1848.6$ \\
\hline White throated fantail & Rhipidura albicollis & 3.8 & $\mathrm{R}(11)$ & 0.004 & W (Sep-Oct) & $1789.7-1966.8$ \\
\hline Kaleej pheasant & Lophura leucomelana & 1.7 & $\mathrm{R}(5)$ & 0.001 & $\begin{array}{c}\text { R, L (Feb-Mar, Apr- May, } \\
\text { Nev) }\end{array}$ & 1848.6-1966.8 \\
\hline
\end{tabular}




\section{International Journal of Environmental Sciences \& Natural Resources}

\begin{tabular}{|c|c|c|c|c|c|c|}
\hline Common koel & Endynamys scolopacea & 21.2 & $\mathrm{C}(61)$ & 0.019 & $\begin{array}{l}\text { R, L (Mar- Apr, May- Jun, Jul- } \\
\text { Aug, Sep-Oct) }\end{array}$ & $1777.5-1876.6$ \\
\hline Eurasian cuckoo & Cuculus canorus & 7.3 & $\mathrm{U}(21)$ & 0.006 & S (Mar- Apr, May- Jun, Jul) & 1841.9-1934.7 \\
\hline Great hill barbet & Megalaima virens & 55.5 & A (160) & 0.051 & $\begin{array}{l}\text { R, L (Feb-Mar, Apr-May, Jun- } \\
\text { Jul, Aug-Sep, Oct-Nev) }\end{array}$ & 1777.5-1966.8 \\
\hline $\begin{array}{l}\text { Black naped green } \\
\text { woodpecker }\end{array}$ & Picus canus & 13.9 & $\mathrm{~F}(40)$ & 0.012 & $\begin{array}{l}\text { R (Jun-Jul, Aug- Sep, Oct- } \\
\text { Nev) }\end{array}$ & $1777.5-1966.8$ \\
\hline $\begin{array}{l}\text { Himalayan pied } \\
\text { woodpecker }\end{array}$ & $\begin{array}{l}\text { Dendrocopus himalay- } \\
\text { ansis }\end{array}$ & 4.5 & $\mathrm{R}(13)$ & 0.004 & S (Jun-Jul, Aug) & $1856.8-1966.8$ \\
\hline $\begin{array}{l}\text { Grey capped pigmy } \\
\text { woodpecker }\end{array}$ & $\begin{array}{l}\text { Dendrocopos canicap- } \\
\text { illus }\end{array}$ & 7.9 & $\mathrm{U}(23)$ & 0.007 & R, L (Jun-Jul, Aug- Sep, Oct) & $1789.7-1966.8$ \\
\hline $\begin{array}{l}\text { Scaly billed wood- } \\
\text { pecker }\end{array}$ & Picus squamatus & 5.2 & $\mathrm{U}(15)$ & 0.004 & W (Sep-Oct, Nev) & 1805.0-1911.7 \\
\hline $\begin{array}{l}\text { Brown fronted wood- } \\
\text { pecker }\end{array}$ & Dendrocopous auriceps & 7.9 & $\mathrm{U}(23)$ & 0.007 & $\begin{array}{l}\text { R, L (Jun-Jul, Aug-Sep, Oct- } \\
\text { Nev) }\end{array}$ & $1777.5-1856.8$ \\
\hline $\begin{array}{l}\text { Fulvous breasted } \\
\text { woodpecker }\end{array}$ & Dendrocopos Macei & 9.7 & $\mathrm{U}(28)$ & 0.008 & S (Jun-Jul, Aug) & 1777.5-1911.7 \\
\hline Speckled piculet & Picumnus innominatus & 5.2 & $\mathrm{U}(15)$ & 0.004 & S (Jun-Jul, Aug) & $1805.0-19911.7$ \\
\hline Indian ring dove & Streptopelia decaocto & 5.2 & $\mathrm{U}(15)$ & 0.004 & S (Jun-Jul, Aug) & 1841.9-1856.8 \\
\hline Oriental turtle dove & Streptopeliaorientallis & 7.3 & $\mathrm{U}(21)$ & 0.007 & S (Jun-Jul, Aug- Sep) & 1848.6-1934.7 \\
\hline $\begin{array}{c}\text { Spotted or Chinese } \\
\text { dove }\end{array}$ & Streptopeliachinensis & 9 & $\mathrm{U}(26)$ & 0.008 & S (Jun-Jul, Aug- Sep) & 1841.9-1966.8 \\
\hline $\begin{array}{l}\text { White breasted } \\
\text { kingfisher }\end{array}$ & Halcyonsmyrnensis & 2.4 & $\mathrm{R}(7)$ & 0.002 & R, L (Jul-Aug, Sep-Oct, Nev) & 1777.5-1789.7 \\
\hline Asian barred owlet & Glaudicium cuculoides & 6.9 & $\mathrm{U}(20)$ & 0.006 & R, L (Jun-Jul, Aug- Sep, Oct) & 1789.7-1966.8 \\
\hline Collared pygmy owlet & Glaudicium brodie & 5.5 & $\mathrm{U}(16)$ & 0.005 & W (Feb-Sep, Nev) & $1777.5-1848.2$ \\
\hline Spotted owlet & Athene brama & 2.1 & $\mathrm{R}(6)$ & 0.001 & W (Feb-Sep, Oct- Nev) & 1777.5-1841.9 \\
\hline Long tailed minivit & Pericrocotus ethologu & 7.3 & $\mathrm{U}(21)$ & 0.006 & S (Sep-Oct) & $1848.6-1966.8$ \\
\hline
\end{tabular}

The families with number of species observed were Turdidae $n=15$, Muscicapidae $n=11$, Timalidae $n=11$, Capitonidae $\mathrm{n}=11$, Passeridae $\mathrm{n}=7$ and Corviidae $\mathrm{n}=7$. In a study of bird of Muzaffarabad city, Awan et al., (2004) presented family wise analysis showing that family Turdidae ( 5 species) dominated the avian fauna followed by Corvidae, Sylviidae (4 species each) and Pycnonotidae (3 species), while minimum numbers were belonging to Passeridae, Campiphagidae, Catheridae and Zosteropodae
(1 species each). Whereas one more study of Awan and Saleem (2007) revealed that family Turdidae was dominant (7 species) followed by Sylviidae (6 species) and Motacillidae and Columbidae (4 species each) in Recreational Park somewhere in same habitat around Muzaffarabad. Family wise Shannon Wiener diversity index was highest for family Corvidae $(0.26)$ followed by Sturnidae, Sylviidae and Estrildidae (0.19) for each and lowest for Remizidae, Phasianidae and Alcedinidae (0.006) for each.

\section{Species richness and abundance}

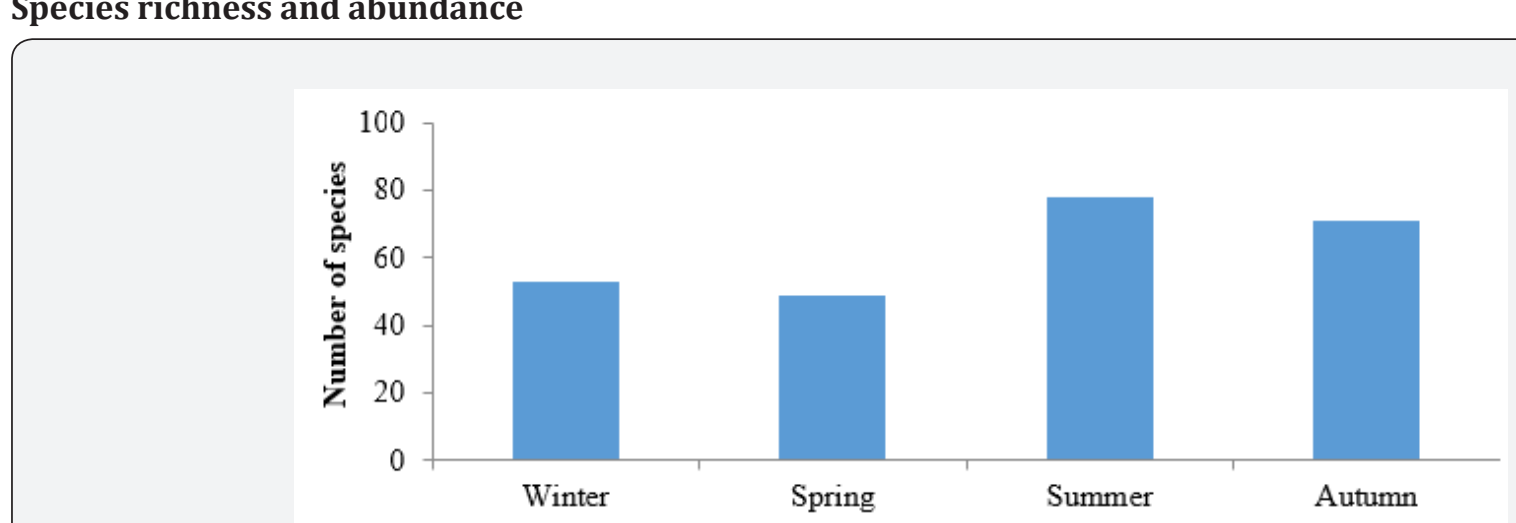

\section{Seasons}

Figure 2: Species richness during different seasons of the year. 
The species richness was estimated to be highest in summer $(\mathrm{n}=78)$, followed by autumn $(\mathrm{n}=72)$, winter $(\mathrm{n}=53)$ and spring $(n=49)$ (Figure 2). Mausad et al. [13] also recorded it to be highest in summer $(n=54)$, followed by autumn $(n=46)$ and spring $(n=40)$ in Chinnari district Hattian, Pakistan within a similar habitat in Kashmir Himalaya. Abundance of birds was also calculated which revealed highest during summer $(n=1148)$, followed by autumn ( $n=863)$, spring $(n=711)$ and winter $(n=415)$ (Figure 2$)$. The availability of fruits and flower nectar in summer may be the reasons of higher abundance in summer. The significant difference in species abundance between different seasons has been recorded ( $p=0.05, F=6.50372)$.

The seasonal changes in the species abundance of birds caused due to divergent seasonality of rainfall and seasonal variation in availability of food resources [16]. The abundance of many bird species is mainly determined by the composition of the vegetation that forms a major element of their habitats. Some changes in vegetation along with biological and environmental gradients causes changes in habitat and a particular bird species can appear, increase or decrease in number and vanish [17].

\section{Seasonal shannon-wiener diversity index}

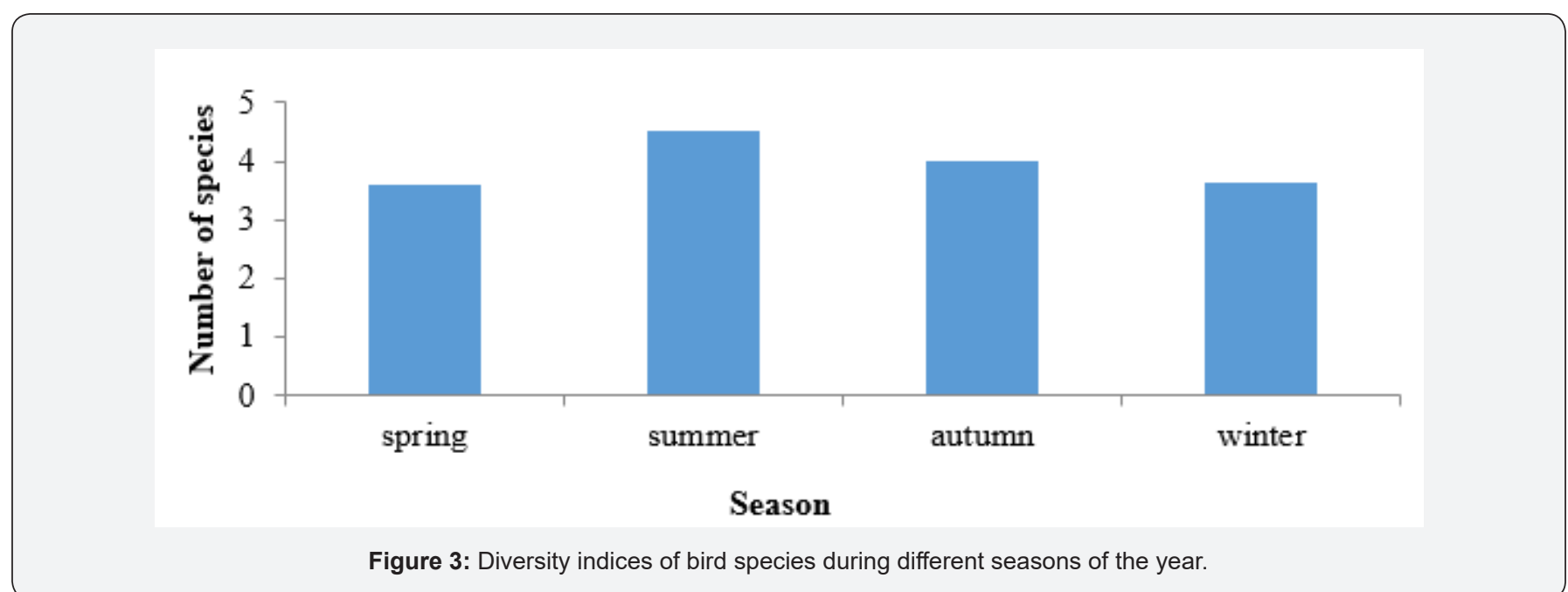

The highest seasonal Shannon index (4.3) of bird species was recorded in summer followed by autumn (3.99), winter (3.64) and spring (3.58) (Figure 3), while its overall value was recorded to (3.9). Faiz et al. [18] surveyed Toli pir national park, district Poonch, Azad Kashmir, Pakistan and calculated overall diversity index (2.22), while Raza et al. [19], reported the avian diversity of Lahore zoo safari, overall diversity index was recorded to be (1.93). Sidra \& Ali [20] during the survey of new campus Punjab university estimated it to be (2.548).

A wide variety of biotic and abiotic factors greatly influenced the diversity and density of bird species. The availability of food might be the main reason for the difference in Population of bird among different seasons during the study duration. As summer is the Prominent breeding season, this might be another reason for high diversity during autumn or early winter the bird species migrates towards lower altitudinal ranges hence the diversity index decreases. The area got heavy snow while fall in winter which prevails during almost half of the spring season, hence a reasonable decrease in diversity index during spring.

\section{Conservation Issues}

The important conservation issues within the Bunjosa game reserve include habitat loss, through collection of timber and firewood, unlawful hunting, overgrazing and pollution.
There is an unlawful falling of trees, as many trees particularly of Pinus wallichiana and Pinus roxburji were recorded cut down by people for fuel wood or construction purpose specially in lower elevation areas of the reserve alongside with residential areas. Indiscriminate deforestation is not merely affecting the habitat of those birds particularly associated with those elevations throughout the year but also those undergo seasonal migration during winter months. To reduce the impacts of harvesting forests it is suggested to clearly marked and monitor the boundaries of the reserve and facilitate local people with some alternate fuel resources [21,22].

The area lies along with residential areas so there is a strong negative interaction of human population with wildlife. People are dependent on natural resources so extensive grazing of domestic animals in forest and grasslands cause destruction and fragmentation of habitats of vital birds' species. In addition, as this area has many grazing lands and remain very attractive for herdsmen and their seasonal migration towards it along with their herds cause considerable damage.

There is a beautiful artificial lake in the center of the reserve which is a famous picnic point as well as a permanent water source for birds. The extensive tourist's activities particularly during summer season badly pollute the lake water and surrounding areas because of the lack of proper sanitation facilities. As there is a 
healthy influx of migratory bird species during summer towards the lake area so they are badly affected by water pollution. In addition to this the area is along with road side and there is a continuous flow of traffic which is considered as a permanent source of disturbance for birds.

As the reserve lies along with road side so it is easily accessible. The fluctuations in habitat and general trends in species richness would be monitored easily to support effective site management. The present study might provide a soul basis to design a bird monitoring scheme in Bunjosa game reserve. In this regard the ordinal categories of relative abundance established previously by Awan et al. [8] in Salkhala game reserve may also be helpful to detect large scale fluctuations in abundance of individual species within the BGR in future. Development of the Conservation Management plan of the game reserve is very important to help protect the biodiversity of the protected area long with unique avian fauna of the area.

\section{References}

1. Bilgrami KS (1995) Concept and Conservation of Biodiversity. CBS Publishers and distributors, Delhi, India.

2. Mian A (1995) Grey partridge demands intensive study. WPA News, 1(2).

3. Chen IC, Hill JK, Ohlemüller R, Roy DB, Thomas CD (2011) Rapid range shifts of species associated with high levels of climate warming. Science 333(6045): 1024-1026.

4. Rapoport EH (1993) The process of plant colonization in small settlements and large cities. In Humans as components of ecosystems. Springer-Verlag, New York, USA, pp. 190-207.

5. Şekercioğlu ÇH, Primack RB, Wormworth J (2012) The effects of climate change on tropical birds. Biological Conservation 148(1): 1-18.

6. Palmer M, Bernehardt E, Chornesky E, Collins S, Dobson A, et al. (2004) Ecology: Ecology for a crowded planet. Science 304(5675): 1251-1252.

7. Bibby CJ, Burgess ND, Hill DA, Mustoe S (2000) Bird census techniques. Second revised edition. Acadmic press, London, UK.

8. Awan MN, Ali H, Lee DC (2012) An annotated checklist of birds and conservation issues in Salkhala Game Reserve, an isolated Important Bird Area in Azad Kashmir, Pakistan. Forktail 28: 38-43.
9. Grimmett R, Roberts T, Inskipp T (2008) A field guide to the birds of Pakistan. Christopher helm London, Yale university press new heaven, USA, p. 241.

10. Mirza ZB, Wasiq H (2007) A field guide to birds of Pakistan, WWF-Pakistan, Bookland Lahore, Pakistan, pp. 10-30.

11. Shannon CE, Weaver W (1963) The Mathematical Theory of Communication. Urbana, I.L: University of Illinois Press, USA, pp. 31-35.

12. Lowen JC, Bartrina L, Clay RP, Tobias JA (1996) Biological surveys and conservation priorities in eastern Paraguay. CSB Cons. Publ, Cambridge, UK.

13. Masaud N, Awan MS, Minhas RA, Ali U (2010) Study of Avian Diversity in and around Chinari, District Hattian, Azad Jammu and Kashmir, Pakistan. Pakistan Journal of Wildlife 1(2): 43-49.

14.Awan MN, Awan AA (2009) Wagtails in Salkhala Game Reserve, Azad Kashmir, Pakistan. Ecology of Berkut 18(1-2): 126-129.

15.Awan MN, Awan MS (2007) Altitudinal range and relative abundance of five species of Tits in Machiara National Park, Muzaffarabad, Azad Kashmir. Ecology of Berkut 16(2): 240-243.

16. Gaston KJ, Blackburn TM, Greenwood JD, Greroryx RD, Rachel MQ, et al. (2000) Abundance-occupancy relationships. Journal of Applied Ecology 37(s1): 39-59.

17.Lee P, Rotenberry JT (2005) Relationships between bird species and tree species assemblages in forested habitats of eastern North America. Journal of Biogeography 32(7): 1139-1150.

18. Faiz AH, Abbas FI, Ali Z, Zahra L (2015) Avifaunal diversity of Toli pir national park Azad Jammu and Kashmir, Pakistan. The Journal of Animal and Plant Sciences 25(3 Supp.2): 404-409.

19. Raza H, Mehmood S, Khan BN, Bibi F, Ali Z (2015) Avian diversity of Lahore zoo safari in winter season Lahore, Pakistan. Journal of Animal and Plant Sciences 25(3 Supp.2): 378-381.

20.Sidra S, Ali Z, Chaudhry MN (2013) Avian diversity at new campus of Punjab University in relation to land use change. Pakistan Journal of Zoology 45(4): 1069-1082.

21. Awan MN, Awan MS. Ahmed KB, Khan AA, Dar NI (2004) A preliminary study on distribution of avian fauna of Muzaffarabad, Azad Jammu and Kashmir. International Journal of Agriculture and Biology 6(2): 300302.

22.Awan MN, Saleem MM (2007) Avifaunal diversity of Pattika recrational park, Muzaffarabad, Azad Kashmir, Pakistan. Zoological Research 28(6): 634-639.

\section{Your next submission with Juniper Publishers will reach you the below assets}

- Quality Editorial service

- Swift Peer Review

- Reprints availability

- E-prints Service

- Manuscript Podcast for convenient understanding

- Global attainment for your research

- Manuscript accessibility in different formats

( Pdf, E-pub, Full Text, Audio)

- Unceasing customer service

Track the below URL for one-step submission https://juniperpublishers.com/online-submission.php 